\title{
Rebuilding Synaptic Architecture in HIV-1 Associated Neurocognitive Disease: A Therapeutic Strategy Based on Modulation of Mixed Lineage Kinase
}

\author{
Harris A. Gelbard, ${ }^{* \dagger}{ }^{\dagger}$ Stephen Dewhurst, ${ }^{\S}$ Sanjay B. Maggirwar, ${ }^{\S}$ Michelle Kiebala, ${ }^{\S}$ \\ Oksana Polesskaya, ${ }^{\S}$ and Howard E. Gendelman ${ }^{\|}$ \\ * Center for Neural Development and Disease, ${ }^{\dagger}$ Department of Neurology (Child Neurology Division), ${ }^{\ddagger}$ Department of Pediatrics, \\ ${ }^{\S}$ Department of Microbiology and Immunology, University of Rochester School of Medicine and Dentistry, Rochester, New York \\ 14642; "Department of Pharmacology and Experimental Neuroscience, University of Nebraska Medical Center, Omaha,
}

Nebraska 68198

\begin{abstract}
Summary:Work from our laboratories has validated mixed lineage kinase type 3 (MLK3) as an enzyme pathologically activated in the CNS by human immunodeficiency virus 1 (HIV-1) neurotoxins. In this review, we discuss MLK3 activation in the context of the neuropathogenesis of HIV-1 associated neurocognitive deficits (HAND). We use findings from the literature to substantiate the neuropathologic relevance of MLK3 to neurodegenerative disease, with an emphasis on Parkinson's disease that shares a number of important phenotypic and neuropathologic characteristics with HAND. We discuss
\end{abstract}

signal transduction pathways downstream from MLK3 activation, with an emphasis on their involvement in microglia and neurons in preclinical models of HAND. Finally, we make a case for pharmacologic intervention targeted at inhibition of MLK3 as a strategy to reverse HAND, in light of the fact that combination antiretroviral therapy, despite successfully managing systemic infection of HIV-1, has been largely unsuccessful in eradicating HAND. Key Words: HIV-1, HIV-1 associated neurocognitive disease (HAND), microglia, mixed lineage kinase type 3 , neuroinflammation, neurotrophins, synapse, tat.

\section{INTRODUCTION}

Combination antiretroviral therapy (cART) has transformed human immunodeficiency virus 1 (HIV-1) infection, once almost uniformly fatal to adults and children, into a chronic, manageable disease. Prior to the advent of cART, HIV-1 infection frequently resulted in a devastating subcortical dementia (HIV-1-associated dementia) in adults and a progressive encephalopathy in children. After the widespread availability of cART, HIV-1-associated dementia and progressive encephalopathy nearly disappeared, but a more indolent phenotype, HIV-associated neurocognitive disorder (HAND) remains a frequent, insidious complication of HIV-1 infection that pervasively diminishes quality of life and overall functionality for the activities of daily living. However, on-

Address correspondence and reprint requests to: Harris A. Gelbard, M.D., Ph.D., Center for Neural Development and Disease, University of Rochester School of Medicine and Dentistry, 601 Elmwood Ave, Box 645, Rochester, NY 14642. E-mail: harris_gelbard@urmc.rochester.edu. going longitudinal and cross-sectional studies of adult and pediatric patients with HIV-1 have shown that efficacious control of viral load with cART has not eradicated the constellation of cognitive neurologic disease that comprises HAND. Current interim estimates of aggregate data from the CNS HIV Anti-Retroviral Therapy Effects Research (CHARTER) consortium suggest that $52 \%$ of their cohort (total $\mathrm{N}=1562$ ) have some form of HAND. ${ }^{1}$ Thus, despite widespread availability and adherence to cART, the prevalence of HAND has actually increased. ${ }^{1,2}$ Moreover, neuropathologic retrospective analyses from patients in the post-cART era have shown a $17.5 \%$ incidence of parenchymal brain pathology, ${ }^{2}$ and cART with increased CNS penetration has been associated with worse neurocognitive performance, ${ }^{3}$ raising the possibility that chronic exposure to cART may have longterm neurotoxic consequences independent of HAND.

More effective forms of cART may be possible through the use of agents capable of reactivating and flushing otherwise stable viral reservoirs. ${ }^{4-6}$ However, the development and clinical testing of new drugs capa- 
ble of reversing HIV-1 latency in the CNS will present unique hurdles and may require considerable time to bring it to fruition. As a result, effective adjunctive therapies for HAND will remain a crucially important unmet medical need for the foreseeable future. This is especially true in light of the lack of efficacy of other candidate adjunctive therapies for HAND, as summarized in a recent meta-analysis of 10 trials of adjunctive therapies involving 711 subjects. $^{7}$ It is worth noting that most of the agents studied in these trials had not been evaluated in an in vivo model to directly test whether the candidate adjunctive therapy was efficacious in reversing either neuroinflammation or restoring synaptic function.

\section{HAND IS A NEUROINFLAMMATORY DISEASE THAT PLACES INCREASED METABOLIC DEMANDS ON THE SYNAPSE}

A 2003 study of HIV-1 infected subjects, previously naïve to antiretroviral therapy, who received 3 months of cART, and subsequently underwent neuropsychologic tests and magnetic resonance spectroscopic (MRS) studies, demonstrated significant improvement in CD4 counts, suppression of plasma, and CSF viral loads. However, the study cohort had persistent elevations of MRS parameters (creatine and choline), which served as markers of inflammation in the frontal lobes. ${ }^{8}$ In addition, neuropsychologic impairments persisted, and creatine and choline increased in the basal ganglia after cART, regardless of whether one or two cART agents effectively penetrated the blood brain barrier. ${ }^{8}$

A year prior to this study, Ernst et al. ${ }^{9}$ showed that there was an increased magnitude and volume of activation measured by blood oxygen level-dependent functional neuroimaging studies in the lateral prefrontal cortex of cognitively normal HIV-1 subjects while performing neuropsychologic tasks of increasing difficulty compared with matched HIV-1 seronegative controls. Seven years later, the authors conducted an equally intriguing blood oxygen level-dependent imaging study during which HIV-1 positive and negative subjects performed tasks of increasing attentional load at an initial trial and a 1-year follow-up trial. ${ }^{10}$ Although both sets of subjects had normal neuropsychologic indices at the initial and follow-up visits, during the follow-up visit, the HIV-1 positive subjects had increased magnitudes of brain activation in the prefrontal and posterior parietal cortex with high-load attentional tasks, whereas the HIV-1 seronegative subjects had decreased magnitudes of brain activation.

Collectively, these studies suggest that HIV-1 infection in the CNS is a chronic neuroinflammatory condition that affects the brain's ability to achieve increased synaptic efficiency, the basis for long-term potentiation that is associated with learning and memory, and to be able to compensate, the brain must activate more polysynaptic networks to achieve normal processing of information. This places increased metabolic demands on the synapse, increasing the potential for permanent damage to synaptic architecture. This also forecasts an increasing neurologic burden for the aging population with HIV-1, who also may be susceptible to developing Alzheimer's and Parkinson's disease (PD), because they will sustain an additive or even synergistic type of neurodegeneration. Thus, we posit that for therapies to be effective at reversing HAND, they must reverse inflammation at the synapse before neuronal function can be ameliorated.

\section{PREVIOUS CLINICAL EXPERIENCE WITH ADJUNCTIVE THERAPIES FOR HAND: WHY DID THEY NOT WORK?}

Development of neuroprotective therapies for neurodegenerative disease face extraordinary challenges, related to the complexity of neuropathogenesis, the bioavailability of drugs in vulnerable brain regions and timing of treatment. For example, Alzheimer's disease and PD likely began many decades before a clinically significant phenotype was noticeable; thus, therapeutic strategies face an uphill battle because damage to vulnerable neuronal pathways may have passed a critical point for repair and return to pre-morbid homeostasis. In contrast, HAND provides some unique opportunities for therapeutic intervention, not least because 1) the onset of HIV-1 infection is usually a definable event that can be routinely tested, and 2) early events in HAND likely involve reversible synaptic injury (see previous).

Notwithstanding, previous trials of adjunctive therapy for HAND have been unsuccessful. As noted previously, a recent meta-analysis of the published outcomes of 10 trials of adjunctive therapies involving 711 people with HIV-1 and neurologic disease concluded there was no evidence for efficacy in terms of cognitive improvement. Although many of these agents had not been evaluated in an appropriate in vivo model of HAND, memantine was a notable exception. Memantine blocks the NMDA receptor-associated ion channel only when it is excessively (i.e., pathologically) open, and as such, does not remain in the channel long enough to block normal excitatory neurotransmission. Despite success in preclinical, small animal models, ${ }^{11}$ memantine failed to significantly improve any neuropsychologic indices in a phase II doubleblind, randomized, placebo-controlled, multi-center trial (plus best anti-retroviral therapy) in patients with HAND. ${ }^{12}$ Interestingly, this was the first clinical study of HIV-associated dementia in which surrogate markers (i.e., MRS) were validated with clinical presentation, as quantified by neurocognitive testing, and in which the surrogate markers appeared to be even more sensitive 
than the cognitive testing or neurologic examination. MRS was used to quantify $\mathrm{N}$-acetylaspartate (NAA), a metabolite that is chiefly found in neurons in the adult brain, and the ratio of NAA to creatine was used as an index of neuronal function and viability. Significant increases in NAA and creatine were observed in the multivariate analysis among individuals receiving memantine compared with the placebo in the frontal white matter and the parietal cortex. Thus, the failure to elicit clinical improvements in neuropsychologic parameters with memantine, despite an increase in the MRS parameter NAA, which was presumably indicative of preserved neuronal integrity in corresponding brain regions, may reflect the fact that neuroinflammation can prevent activitydependent (i.e., long-term potentiation) processes, both upstream and downstream of the NMDA-R channel. ${ }^{13-15}$

This emphasizes the need to design adjunctive therapies that take into account both neuroinflammation caused by HIV-1 viral products and pro-inflammatory mediators, and the activity-dependent nature of neuronal networks vulnerable to these neurotoxins, rather than simply quantifying neuronal apoptosis or even synaptic transmission as a therapeutic endpoint.

\section{MIXED LINEAGE KINASE}

Mixed-lineage kinase (MLK) are mitogen-activated protein kinase (MAPK) kinase kinases (MKKKs) with features of both serine-threonine and tyrosine kinases (hence the nomenclature "mixed lineage") that regulate the c-Jun Nterminal kinase (JNK) MAPK signaling cascade, and also that regulate the other two major MAPK pathways, p38 and extracellular signal-regulated kinase. ${ }^{16-18}$

MLK3 (also known as MAP3K11) is the most widely expressed MLK family member, ${ }^{16-18}$ and is expressed in neurons, ${ }^{19}$ dendritic cells, ${ }^{20,21}$ and many other cell types. At the cellular level, MLK3 is activated by cellular and metabolic stress, including reactive oxygen species, ceramide, and tumor necrosis factor- $\alpha .^{22,23}$ At the molecular level, MLK3 is activated by the small GTPases, Cdc42, and Rac, which bind to the interactive binding region of MLK3, and can cause it to homodimerize via a leucine zipper interface, resulting in autophosphorylation at Thr277 and Ser281 within the protein activation loop, ultimately resulting in MLK3 activation. ${ }^{24,25}$ HIV-1 Tat also leads to phosphorylation at these same residues, with accompanying activation of MLK3 in primary rat neurons. ${ }^{26}$

\section{MLK3 AS A THERAPEUTIC TARGET IN NEURODEGENERATIVE DISEASE}

MLK3 has been implicated in neuronal apoptosis leading to neurodegenerative disease. ${ }^{26-29}$ In the context of PD, the first-generation MLK3 inhibitor, Cephalon (CEP)-1347, has been shown to prevent the induction of neuronal cell death, motor deficits, and neuronal degeneration in the 1-methyl-4phenyl-1,2,3,6-tetrahydropyridine (MPTP) model of Parkinsonism, ${ }^{30-33}$ and CEP1347-mediated neuroprotection has also been demonstrated in an in vitro model for PD, using methamphetamine-exposed human mesencephalic-derived neurons. ${ }^{30}$ In addition, MLK3 has been implicated as playing a causal role in peripheral neuronal degeneration, including the development of HIV-associated peripheral neuropathy, which can be induced both by soluble HIV-1 gene products and also by the anti-viral drugs used to treat HIV-1.34,35

The results of a phase II trial of CEP1347 for treatment of PD were reported in 2007. This trial, the Parkinson Research Examination CEP-1347 Trial (PRECEPT), enrolled PD patients in the early stages of disease, not yet requiring dopaminergic therapy $(\mathrm{N}=806){ }^{36}$ The primary endpoint was time to disability requiring dopaminergic replacement therapy with L-dopa. Unfortunately, the study was concluded early due to futility, ${ }^{36}$ raising questions about the appropriateness of the PD models used in preclinical studies with CEP-1347, ${ }^{37}$ as well as about the CNS pharmacokinetics, target selectivity, and mechanism of action of CEP-1347 itself. $^{38}$ Wang and Johnson $^{39}$ surmised that failure of the Parkinson Research Examination CEP-1347 Trial was due to the fact that CEP-1347 inhibition of MLK3 in dopaminergic neurons was insufficient to ensure survival without additional trophic support from brain-derived neurotrophic factor (BDNF) signaling via TrkB receptors.

\section{MLK3 AS A THERAPEUTIC TARGET IN HAND}

In vitro studies of Cephalon's "first generation" MLK inhibitor, CEP1347, showed that this agent can protect primary rat hippocampal neurons as well as dorsal root ganglion neurons from the otherwise lethal effects of exposure to HIV-1 gp120. ${ }^{34,35}$ Studies from our laboratories subsequently examined the effect of HIV-1 Tat and gp120 on MLK3. ${ }^{26}$ Tat and gp120 were shown to induce autophosphorylation of MLK3 in primary rat neurons and this was abolished by the addition of CEP1347. ${ }^{26}$ The use of CEP1347 also enhanced survival of both rat and human neurons and inhibited the activation of human monocytes after exposure to Tat and gp $120 .^{26}$ Furthermore, overexpression of wild-type MLK3 led to the induction of neuronal death, whereas expression of a dominant negative MLK3 mutant protected neurons from the toxic effects of Tat. ${ }^{26}$

We further confirmed and extended these in vitro findings by showing that CEP1347 is neuroprotective in an in vivo model of HIV-1 infection, reversing microglial activation and restoring normal synaptic architecture, as well as restoring macrophage secretory profiles to a trophic versus toxic phenotype in response to HIV-1 infec- 
A

\section{HIV-1 infection}

\section{HAND}

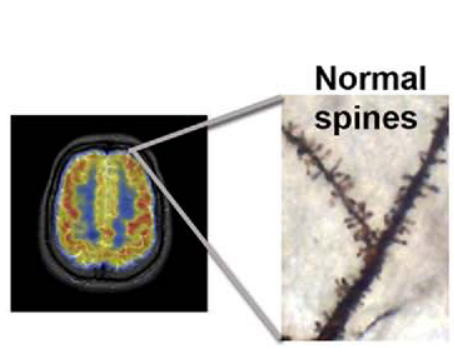

scant leukocyte infiltration
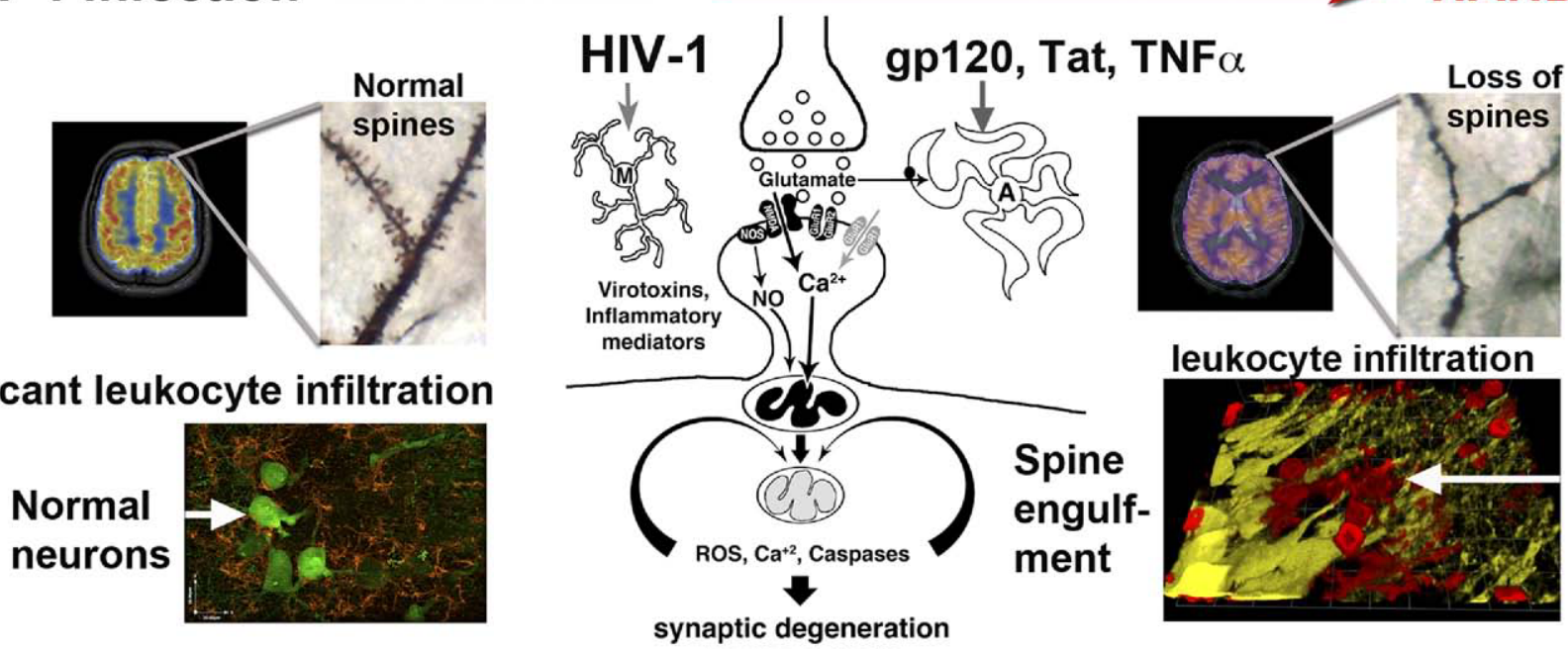

neuronal death

B

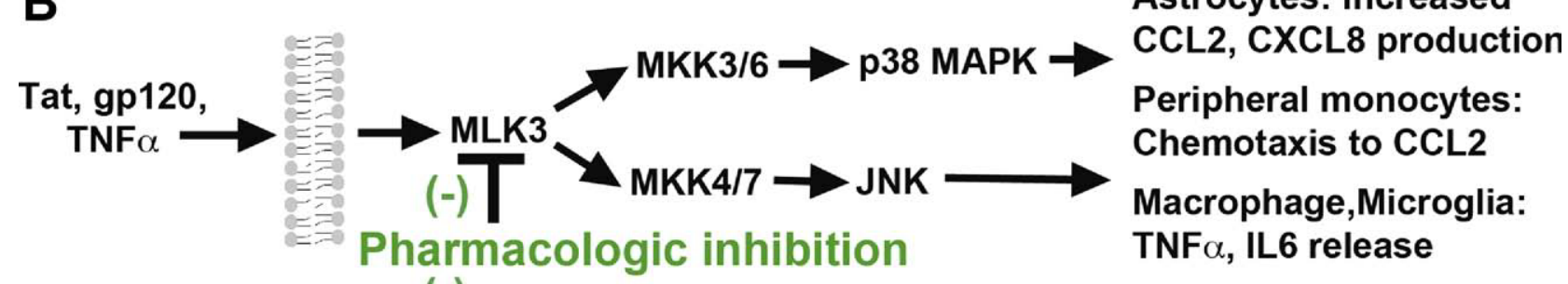

Astrocytes: Increased CCL2, CXCL8 production

Peripheral monocytes: Chemotaxis to CCL2

TNF $\alpha$, IL6 release

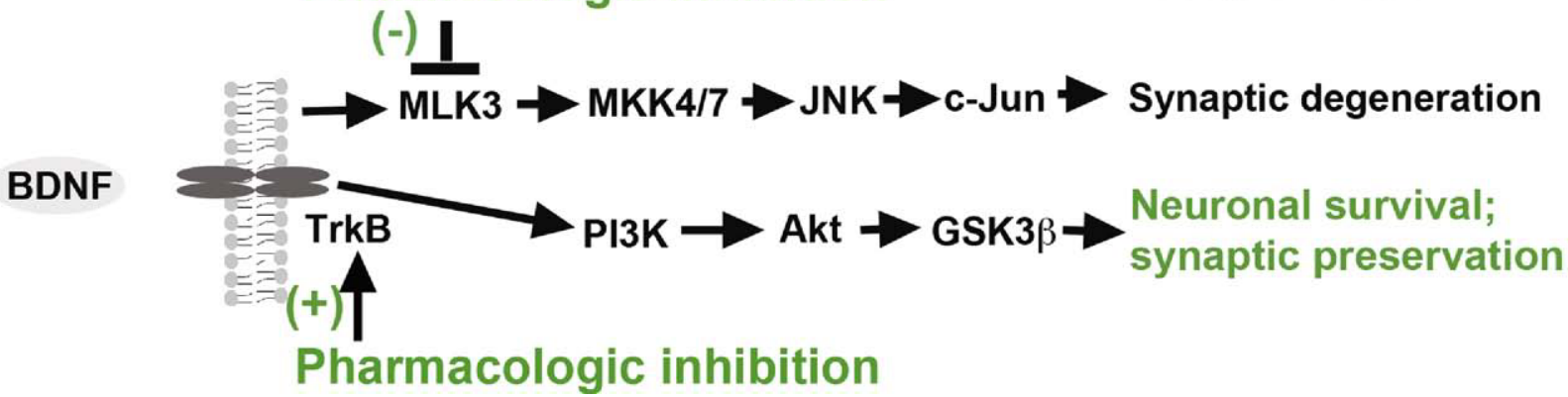

FIG. 1. Neuropathogenesis of human immunodeficiency virus (HIV)-1 associated neurocognitive deficits (HAND) and critical mixed lineage kinase type 3 (MLK3) pathways. (A) (Top left): fMRI scan of an HIV patient without HAND and a representative postmortem Golgi stain of intact dendrites and spines from the frontal cortex. (Top right): fMRI scan from an HIV patient with HAND and a representative Golgi stain of dendritic beading with atrophic or absent spines. (Center): Pre- and post-synaptic elements (terminal bouton and dendritic spine, respectively) of a synapse with an infiltrating HIV-1 infected mononuclear phagocyte (M) and an astrocyte (A) with normal glutamate uptake/transport impeded by gp120, Tat, and tumor necrosis factor (TNF)- $\alpha$. (Bottom left): Normal hippocampal neurons expressing enhanced green fluorescent protein (eGFP, white arrow) and CD11b+ microglia with little or no evidence of infiltrating leukocytes 1 day after stereotactic injection of control vehicle. (Bottom right): Damaged hippocampal neurons expressing eGFP (pseudo-colored yellow for clarity) and CD11b + infiltrating inflammatory leukocytes engulfing neuronal dendritic spines (white arrow) 1 day after stereotactic injection of HIV-1 Tat. (B) (Top): MLK3 intracellular signaling pathways involving chemokine and cytokine activation in HAND. (Bottom): MLK3 and disruption of neurotrophin homeostasis in HAND.

tion. ${ }^{40}$ Collectivcely, these studies suggest that MLK3 activity is increased by HIV-1 neurotoxins, resulting in downstream signaling events that trigger neuronal death and damage, along with monocyte activation (accompanied by release of inflammatory cytokines). We have delineated some of the key features of MLK3 activation in the cartoon (FIGS. 1A and 1B) as follows.

\section{MLK3 SIGNALING EVENTS RELEVANT TO HAND: 1) MONOCYTE CHEMOTAXIS AND NEUROINFLAMMATION}

Monocyte recruitment to the CNS has long been recognized as an important contributor to viral neuro-invasion, and macrophage burden in the CNS represents a 
strong correlate of HIV-associated neurologic disease. ${ }^{41}$ Many of these macrophage express activation markers and are believed to originate from peripheral monocytes, including subsets of monocytes with inflammatory characteristics that have been associated with the clinical presentation of HIV-1 or SIV encephalitis. ${ }^{42-48}$ Importantly, infiltration of peripheral monocytes and their persistent activation in certain brain regions, such as hippocampus and adjacent parts of entorhinal and temporal cortex persists in cART-treated HIV-1 infected patients. ${ }^{49-52}$ Thus, monocyte chemotaxis into the CNS is believed to play an important role in the neuropathogenesis of HAND.

Expression of the potent monocyte chemokine, CCL2 (also known as MCP1) is upregulated by HIV-1 Tat, ${ }^{53-65}$ and is elevated in brains from subjects with HIV-1 dementia. ${ }^{54}$ Interestingly, CCL2-mediated monocyte chemotaxis is synergistically enhanced by CXCL8 (also known as interleukin-8), ${ }^{65-67}$ which is also upregulated by HIV-1 transactivator of transcription (Tat). ${ }^{55,65}$ These obscervations are relevant to MLK3 signaling events because astrocytic expression of CCL2 and CXCL8 is regulated by JNK, ${ }^{37,65,68-72}$ and JNK lies downstream of MLK3 (FIG. 1B). Moreover, CCL2 engagement of its cognate receptor, CCR2, results in activation of downstream MLK3 signaling events, including activation of the JNK and p38 MAPK pathways. ${ }^{62,73,74}$ Thus, MLK3 regulated signaling cascades likely play an important role in monocyte chemotaxis to the CNS, via increased CCL2 and CXCL8 production from astrocytes (FIG. 1B).

MLK3 also modulates neuroinflammation through effects on cytokine production by microglia and brain macrophages. In particular, CEP1347-mediated inhibition of MLK3 results in a significant decrease in Tat-stimulated release of tumor necrosis factor alpha (TNF- $\alpha$ ) and interleukin- 6 by macrophage and microglia. ${ }^{75}$ This is important because TNF- $\alpha$ mediates a significant part of Tat-mediated toxicity in models of HAND, ${ }^{76}$ and inhibition of this pathway by CEP-1347 may account for the reduction in neuroinflammation in an in vivo model for HAND. ${ }^{40}$

\section{MLK3 SIGNALING EVENTS RELEVANT TO HAND: 2) SYNAPTIC HOMEOSTASIS AND NEUROTROPHIN SIGNALING}

There is another, equally important aspect to the role of MLK3 inhibition as a potential therapeutic strategy for HAND: its role is in promoting neurotrophin signaling and synaptic homeostasis (FIG. 1B). These processes may be coupled because trophic factor production by microglia (e.g., release of BDNF) may be reduced in response to the presence of infiltrating leukocytes within the CNS, leading to a reduction in trophic support for
TrkB bearing neurons. ${ }^{77-80}$ Moreover, changes in neurotrophin signaling may affect microglial activation, but the mechanisms for this are complex because microglia express truncated TrkB receptors (Maggirwar et al., unpublished data) and respond to neurotrophins (including BDNF), but are likely to have different downstream signaling repertoires than neurons that express full-length TrkB receptors. Indeed, BDNF inhibits nitric oxide release by activated microglia, suggesting that it may have a direct anti-neuro-inflammatory effect. ${ }^{81,82}$

MLK regulates Trk receptor expression at the transcriptional level, ${ }^{83}$ and MLK3 inhibition results in a robust up-regulation of TrkB expression. ${ }^{83,84}$ Moreover, ongoing studies in our laboratories demonstrate that pharmacologic inhibition of MLK3 is also able to increase TrkB expression in primary neurons (data not shown), suggesting that this inhibition may potentiate neutrophin signaling.

Finally, there is evidence that the HIV-1 envelope protein gp120 reduces levels of BDNF in vivo, ${ }^{85}$ presumably decreasing its ability to activate TrkB receptors and extracellular signal-regulated kinase 1/2-mediated neuroprotective pathways. ${ }^{86}$ Thus, gp120 induces neurotoxicity in part by its ability to interfere with BDNF signaling via TrkB and activates apoptotic pathways by a caspase-3-dependent mechanism. ${ }^{86}$ The overall contribution of gp120 versus Tat to the pathogenesis of HAND, as well as the relative overlap in MLK3 and BDNFassociated signaling pathways, still remain active areas of investigation.

\section{CONCLUSIONS}

In summary, our studies underscore the importance of MLK3 as a therapeutic target in HIV-associated neurocognitive disorder. In particular, our data implicate microglia and inflammatory leukocytes infiltrating into the CNS as key targets for MLK3 inhibition to prevent neuro-inflammation and destruction of vulnerable synaptic architecture. This type of therapeutic strategy is necessary to address the ongoing neuro-inflammation that persists in the face of successful resolution of systemic HIV-1 infection by cART. Our data also suggest a compelling need for next generation pharmacologic approaches to inhibit MLK3 and to elaborate the identification of MLK3-based neuroprotective mechanisms.

Acknowledgments: We are grateful for the support of the National Institutes of Health awards No. PO1 MH64570 (HAG, SD, SBM, HEG), No. RO1 MH56838 (HAG), No. RO1 MH078989 (HAG), No. R21 MH03851 (HAG), No. RO1 DA026325 (SD), No. RO1 NS054578 (SBM), No. T32 AI49105 (MK), and the generous support of the Geoffrey Waasdorp Pediatric Neurology Fund without which this work would not have been possible. 


\section{REFERENCES}

1. Heaton R, Clifford D, Woods S, et al. HIV-associated neurocognitive impairment remains prevalent in the era of combination ART: The CHARTER Study. Paper presented at: 16th Conference on Retroviruses and Opportunistic Infections; February 2009; Montreal, CA.

2. Neuenburg JK, Brodt HR, Herndier BG, et al. HIV-related neuropathology, 1985 to 1999: rising prevalence of HIV encephalopathy in the era of highly active antiretroviral therapy. J Acquir Immune Defic Syndr 2002;31:171-177.

3. Marra CM, Zhao Y, Clifford DB, et al. Impact of combination antiretroviral therapy on cerebrospinal fluid HIV RNA and neurocognitive performance. Aids 2009;23:1359-1366.

4. Brennan TP, Woods JO, Sedaghat AR, Siliciano JD, Siliciano RF, Wilke CO. Analysis of human immunodeficiency virus type 1 viremia and provirus in resting $\mathrm{CD} 4+\mathrm{T}$ cells reveals a novel source of residual viremia in patients on antiretroviral therapy. J Virol 2009;83:8470-8481.

5. Dinoso JB, Rabi SA, Blankson JN, et al. A simian immunodeficiency virus-infected macaque model to study viral reservoirs that persist during highly active antiretroviral therapy. J Virol 2009;83: 9247-9257.

6. Yang HC, Xing S, Shan L, et al. Small-molecule screening using a human primary cell model of HIV latency identifies compounds that reverse latency without cellular activation. J Clin Invest 2009; 119:3473-3486.

7. Uthman OA, Abdulmalik JO. Adjunctive therapies for AIDS dementia complex. Cochrane Database Syst Rev 2008:CD006496.

8. Chang L, Ernst T, Witt MD, et al. Persistent brain abnormalities in antiretroviral-naive HIV patients 3 months after HAART. Antivir Ther 2003;8:17-26.

9. Ernst T, Chang L, Jovicich J, Ames N, Arnold S. Abnormal brain activation on functional MRI in cognitively asymptomatic HIV patients. Neurology 2002;59:1343-1349.

10. Ernst T, Yakupov R, Nakama H, et al. Declined neural efficiency in cognitively stable human immunodeficiency virus patients. Ann Neurol 2009;65:316-325.

11. Anderson ER, Gendelman HE, Xiong H. Memantine protects hippocampal neuronal function in murine human immunodeficiency virus type 1 encephalitis. J Neurosci 2004;24:7194-7198.

12. Schifitto G, Navia BA, Yiannoutsos CT, et al. Memantine and HIV-associated cognitive impairment: a neuropsychological and proton magnetic resonance spectroscopy study. Aids 2007;21: $1877-1886$.

13. Bellizzi MJ, Lu SM, Gelbard HA. Protecting the synapse: evidence for a rational strategy to treat HIV-1 associated neurologic disease. J Neuroimmune Pharmacol 2006;1:20-31.

14. Bellizzi MJ, Lu SM, Masliah E, Gelbard HA. Synaptic activity becomes excitotoxic in neurons exposed to elevated levels of platelet-activating factor. J Clin Invest 2005;115:3185-3192.

15. Ellis R, Langford D, Masliah E. HIV and antiretroviral therapy in the brain: neuronal injury and repair. Nat Rev Neurosci 2007;8: $33-44$.

16. Gallo KA, Johnson GL. Mixed-lineage kinase control of JNK and p38 MAPK pathways. Nat Rev Mol Cell Biol 2002;3:663-672.

17. Silva RM, Kuan CY, Rakic P, Burke RE. Mixed lineage kinasec-jun N-terminal kinase signaling pathway: a new therapeutic target in Parkinson's disease. Mov Disord 2005;20:653-664.

18. Wang LH, Besirli CG, Johnson EM Jr. Mixed-lineage kinases: a target for the prevention of neurodegeneration. Annu Rev Pharmacol Toxicol 2004;44:451-474.

19. Maroney AC, Finn JP, Connors TJ, et al. Cep-1347 (KT7515), a semisynthetic inhibitor of the mixed lineage kinase family. J Biol Chem 2001;276: 25302-25308.

20. Handley ME, Rasaiyaah J, Barnett J, et al. Expression and function of mixed lineage kinases in dendritic cells. Int Immunol 2007;19: 923-933.

21. Handley ME, Rasaiyaah J, Chain BM, Katz DR. Mixed lineage kinases (MLKs): a role in dendritic cells, inflammation and immunity? Int J Exp Pathol 2007;88:111-126.

22. Jaeschke A, Davis RJ. Metabolic stress signaling mediated by mixed-lineage kinases. Mol Cell 2007;27:498-508.
23. Sathyanarayana P, Barthwal MK, Kundu CN, et al. Activation of the drosophila MLK by ceramide reveals TNF-alpha and ceramide as agonists of mammalian MLK3. Mol Cell 2002;10:1527-1533.

24. Leung IW, Lassam N. Dimerization via tandem leucine zippers is essential for the activation of the mitogen-activated protein kinase kinase kinase, MLK-3. J Biol Chem 1998;273:32408-32415.

25. Leung IW, Lassam N. The kinase activation loop is the key to mixed lineage kinase-3 activation via both autophosphorylation and hematopoietic progenitor kinase 1 phosphorylation. J Biol Chem 2001;276:1961-1967.

26. Sui Z, Fan S, Sniderhan L, et al. Inhibition of mixed lineage kinase 3 prevents HIV-1 Tat-mediated neurotoxicity and monocyte activation. J Immunol 2006;177:702-711.

27. Mishra R, Barthwal MK, Sondarva G, et al. Glycogen synthase kinase-3beta induces neuronal cell death via direct phosphorylation of mixed lineage kinase 3. J Biol Chem 2007;282:30393-30405.

28. Mota M, Reeder M, Chernoff J, Bazenet CE. Evidence for a role of mixed lineage kinases in neuronal apoptosis. J Neurosci 2001; 21:4949-4957.

29. Savinainen A, Garcia EP, Dorow D, Marshall J, Liu YF. Kainate receptor activation induces mixed lineage kinase-mediated cellular signaling cascades via post-synaptic density protein 95 . J Biol Chem 2001;276:11382-11386.

30. Lotharius J, Falsig J, van Beek J, et al. Progressive degeneration of human mesencephalic neuron-derived cells triggered by dopamine-dependent oxidative stress is dependent on the mixed-lineage kinase pathway. J Neurosci 2005;25:6329-6342.

31. Mathiasen JR, McKenna BA, Saporito MS, et al. Inhibition of mixed lineage kinase 3 attenuates MPP+-induced neurotoxicity in SH-SY5Y cells. Brain Res 2004;1003:86-97.

32. Saporito MS, Brown EM, Miller MS, Carswell S. CEP-1347/KT7515 , an inhibitor of c-jun N-terminal kinase activation, attenuates the 1-methyl-4-phenyl tetrahydropyridine-mediated loss of nigrostriatal dopaminergic neurons in vivo. J Pharmacol Exp Ther 1999; 288:421-427.

33. Saporito MS, Hudkins RL, Maroney AC. Discovery of CEP-1347/ KT-7515, an inhibitor of the JNK/SAPK pathway for the treatment of neurodegenerative diseases. Prog Med Chem 2002;40:23-62.

34. Bodner A, Maroney AC, Finn JP, Ghadge G, Roos R, Miller RJ. Mixed lineage kinase 3 mediates gp120IIIB-induced neurotoxicity. J Neurochem 2002;82:1424-1434.

35. Bodner A, Toth PT, Miller RJ. Activation of c-Jun N-terminal kinase mediates gp120IIIB- and nucleoside analogue-induced sensory neuron toxicity. Exp Neurol 2004;188:246-253.

36. Parkinson Study Group PRECEPT Investigators. Mixed lineage kinase inhibitor CEP-1347 fails to delay disability in early Parkinson disease. Neurology 2007;69:1480-1490.

37. Wang J, Gigliotti F, Bhagwat SP, Maggirwar SB, Wright TW Pneumocystis stimulates MCP-1 production by alveolar epithelial cells through a JNK-dependent mechanism. Am J Physiol Lung Cell Mol Physiol 2007;292:L1495-L1505.

38. Saporito MS, Thomas BA, Scott RW. MPTP activates c-Jun $\mathrm{NH}(2)$-terminal kinase (JNK) and its upstream regulatory kinase MKK4 in nigrostriatal neurons in vivo. J Neurochem 2000;75: $1200-1208$.

39. Wang LH, Johnson EM Jr. Mixed lineage kinase inhibitor CEP1347 fails to delay disability in early Parkinson disease. Neurology 2008;71:462-463.

40. Eggert D, Dash PK, Gorantla S, et al. Neuroprotective activities of CEP-1347 in models of neuroAIDS. J Immunol 2010;184:746-756.

41. Glass JD, Fedor H, Wesselingh SL, McArthur JC. Immunocytochemical quantitation of human immunodeficiency virus in the brain: correlations with dementia. Ann Neurol 1995;38:755-762.

42. Borda JT, Alvarez X, Mohan M, et al. CD163, a marker of perivascular macrophages, is up-regulated by microglia in simian immunodeficiency virus encephalitis after haptoglobin-hemoglobin complex stimulation and is suggestive of breakdown of the bloodbrain barrier. Am J Pathol 2008;172:725-737.

43. Fabriek BO, Van Haastert ES, Galea I, et al. CD163-positive perivascular macrophages in the human CNS express molecules for antigen recognition and presentation. Glia 2005;51:297-305.

44. Kim WK, Alvarez X, Fisher J, et al. CD163 identifies perivascular macrophages in normal and viral encephalitic brains and potential 
precursors to perivascular macrophages in blood. Am J Pathol 2006; $168: 822-834$.

45. Marcondes MC, Lanigan CM, Burdo TH, Watry DD, Fox HS. Increased expression of monocyte CD44v6 correlates with the deveopment of encephalitis in rhesus macaques infected with simian immunodeficiency virus. J Infect Dis 2008;197:1567-1576.

46. Pulliam L, Gascon R, Stubblebine M, Mcguire D, McGrath MS. Unique monocyte subset in patients with AIDS dementia. Lancet 1997;349:692-695.

47. Roberts ES, Masliah E, Fox HS. CD163 identifies a unique population of ramified microglia in HIV encephalitis (HIVE). J Neuropathol Exp Neurol 2004;63:1255-1264.

48. Roberts ES, Zandonatti MA, Watry DD, et al. Induction of pathogenic sets of genes in macrophages and neurons in NeuroAIDS. Am J Pathol 2003;162:2041-2057.

49. Anthony IC and Bell JE. The Neuropathology of HIV/AIDS. Int Rev Psychiatry 2008;20:15-24.

50. Anthony IC, Ramage SN, Carnie FW, Simmonds P, Bell JE. Influence of HAART on HIV-related CNS disease and neuroinflammation. J Neuropathol Exp Neurol 2005;64:529-536.

51. Everall IP, Hansen LA, Masliah E. The shifting patterns of HIV encephalitis neuropathology. Neurotox Res 2005;8:51-61.

52. Gray F, Chretien F, Vallat-Decouvelaere AV, Scaravilli F. The changing pattern of HIV neuropathology in the HAART era. J Neuropathol Exp Neurol 2003;62:429-440.

53. Abraham S, Sawaya BE, Safak M, Batuman O, Khalili K, Amini S. Regulation of MCP-1 gene transcription by Smads and HIV-1 Tat in human glial cells. Virology 2003;309:196-202.

54. Conant K, Garzino-Demo A, Nath A, et al. Induction of monocyte chemoattractant protein-1 in HIV-1 Tat-stimulated astrocytes and elevation in AIDS dementia. Proc Natl Acad Sci U S A 1998;95: 3117-3121.

55. D'Aversa TG, Yu KO, Berman JW. Expression of chemokines by human fetal microglia after treatment with the human immunodeficiency virus type 1 protein Tat. J Neurovirol 2004;10:86-97.

56. El-Hage N, Wu G, Ambati J, Bruce-Keller AJ, Knapp PE, Hauser KF. CCR2 mediates increases in glial activation caused by exposure to HIV-1 Tat and opiates. J Neuroimmunol 2006;178:9-16.

57. El-Hage N, Wu G, Wang J, et al. HIV-1 Tat and opiate-induced changes in astrocytes promote chemotaxis of microglia through the expression of MCP-1 and alternative chemokines. Glia 2006;53: 132-146.

58. Eugenin EA, D’Aversa TG, Lopez L, Calderon TM, Berman JW. MCP-1 (CCL2) protects human neurons and astrocytes from NMDA or HIV-tat-induced apoptosis. J Neurochem 2003;85:1299-1311.

59. Eugenin EA, Dyer G, Calderon TM, Berman JW. HIV-1 tat protein induces a migratory phenotype in human fetal microglia by a CCL2 (MCP-1)-dependent mechanism: possible role in NeuroAIDS. Glia 2005;49:501-510.

60. Lim SP, Garzino-Demo A. The human immunodeficiency virus type 1 Tat protein up-regulates the promoter activity of the betachemokine monocyte chemoattractant protein 1 in the human astrocytoma cell line U-87 MG: role of SP-1, AP-1, and NF-kappaB consensus sites. J Virol 2000;74:1632-1640.

61. McManus CM, Weidenheim K, Woodman SE, et al. Chemokine and chemokine-receptor expression in human glial elements: induction by the HIV protein, Tat, and chemokine autoregulation. Am J Pathol 2000;156:1441-1453.

62. Sodhi A, Biswas SK. Monocyte chemoattractant protein-1-induced activation of p42/44 MAPK and c-Jun in murine peritoneal macrophages: a potential pathway for macrophage activation. J Interferon Cytokine Res 2002;22:517-526.

63. Toborek M, Lee YW, Pu H, et al. HIV-Tat protein induces oxidative and inflammatory pathways in brain endothelium. J Neurochem 2003;84:169-179.

64. Weiss JM, Nath A, Major EO, Berman JW. HIV-1 Tat induces monocyte chemoattractant protein-1-mediated monocyte transmigration across a model of the human blood-brain barrier and upregulates CCR5 expression on human monocytes. J Immunol 1999;163:2953-2959.

65. Zheng JC, Huang Y, Tang K, et al. HIV-1-infected and/or immuneactivated macrophages regulate astrocyte CXCL8 production through
IL-1beta and TNF-alpha: involvement of mitogen-activated protein kinases and protein kinase R. J Neuroimmunol 2008;200:100-10.

66. Gouwy M, Struyf S, Noppen S, et al. Synergy between coproduced $\mathrm{CC}$ and CXC chemokines in monocyte chemotaxis through receptor-mediated events. Mol Pharmacol 2008;74:485-495.

67. Gouwy M, Struyf S, Verbeke H, et al. CC chemokine ligand-2 synergizes with the nonchemokine $\mathrm{G}$ protein-coupled receptor ligand fMLP in monocyte chemotaxis, and it cooperates with the TLR ligand LPS via induction of CXCL8. J Leukoc Biol 2009;86: 671-680.

68. Ahmed RA, Murao K, Imachi H, et al. c-Jun N-terminal kinases inhibitor suppresses the TNF-alpha induced MCP-1 expression in human umbilical vein endothelial cells. Endocrine 2009;35:184-188.

69. Arndt PG, Suzuki N, Avdi NJ, Malcolm KC, Worthen GS. Lipopolysaccharide-induced c-Jun NH2-terminal kinase activation in human neutrophils: role of phosphatidylinositol 3-Kinase and Sykmediated pathways. J Biol Chem 2004; 279:10883-10891.

70. Gao YJ, Zhang L, Samad OA, et al. JNK-induced MCP-1 production in spinal cord astrocytes contributes to central sensitization and neuropathic pain. J Neurosci 2009;29:4096-4108.

71. Wolter S, Doerrie A, Weber A, et al. c-Jun controls histone modifications, NF-kappaB recruitment, and RNA polymerase II function to activate the ccl2 gene. Mol Cell Biol 2008;28:4407-4423.

72. Young SK, Arndt PG. c-Jun NH2-terminal kinase regulates lipopolysaccharide-induced pulmonary mononuclear cell recruitment via CCL2. Exp Lung Res 2009;35:682-700.

73. Cambien B, Pomeranz M, Millet MA, Rossi B, Schmid-Alliana A. Signal transduction involved in MCP-1-mediated monocytic transendothelial migration. Blood 2001;97:359-366.

74. Werle M, Schmal U, Hanna K, Kreuzer J. MCP-1 induces activation of MAP-kinases ERK, JNK and p38 MAPK in human endothelial cells. Cardiovasc Res 2002;56:284-292.

75. Sui Z, Kovacs AD, Maggirwar SB. Recruitment of active glycogen synthase kinase-3 into neuronal lipid rafts. Biochem Biophys Res Commun 2006;345:1643-1648.

76. New DR, Maggirwar SB, Epstein LG, Dewhurst S, Gelbard HA. HIV-1 Tat induces neuronal death via tumor necrosis factor-alpha and activation of non-N-methyl-D-aspartate receptors by a NFkappaBindependent mechanism. J Biol Chem 1998;273:17852-17858.

77. Asami T, Ito T, Fukumitsu H, Nomoto H, Furukawa Y, Furukawa $\mathrm{S}$. Autocrine activation of cultured macrophages by brain-derived neurotrophic factor. Biochem Biophys Res Commun 2006;344: 941-947.

78. Batchelor PE, Liberatore GT, Wong JY, et al. Activated macrophages and microglia induce dopaminergic sprouting in the injured striatum and express brain-derived neurotrophic factor and glial cell line-derived neurotrophic factor. J Neurosci 1999;19:1708-1716.

79. Lai AY, Todd KG. Differential regulation of trophic and proinflammatory microglial effectors is dependent on severity of neuronal injury. Glia 2008;56:259-270.

80. Nakajima K, Honda S, Tohyama Y, Imai Y, Kohsaka S, Kurihara T. Neurotrophin secretion from cultured microglia. J Neurosci Res 2001;65:322-331.

81. Mizoguchi Y, Monji A, Kato T, et al. Brain-derived neurotrophic factor induces sustained elevation of intracellular $\mathrm{Ca} 2+$ in rodent microglia. J Immunol 2009;183:7778-7786.

82. Nakajima K, Kikuchi Y, Ikoma E, et al. Neurotrophins regulate the function of cultured microglia. Glia 1998;24:272-289.

83. Pedraza N, Rafel M, Navarro I, Encinas M, Aldea M, Gallego C. Mixed lineage kinase phosphorylates transcription factor E47 and inhibits TrkB expression to link neuronal death and survival pathways. J Biol Chem 2009;284:32980-32988.

84. Wang LH, Paden AJ, Johnson EM Jr. Mixed-lineage kinase inhibitors require the activation of Trk receptors to maintain long-term neuronal trophism and survival. J Pharmacol Exp Ther 2005;312: 1007-1019.

85. Nosheny RL, Ahmed F, Yakovlev A, et al. Brain-derived neurotrophic factor prevents the nigrostriatal degeneration induced by human immunodeficiency virus-1 glycoprotein 120 in vivo. Eur J Neurosci 2007;25:2275-2284.

86. Mocchetti I, Bachis A. Brain-derived neurotrophic factor activation of TrkB protects neurons from HIV-1/gp120-induced cell death. Crit Rev Neurobiol 2004;16:51-57. 